\title{
Vortrag über ein kyphotisch-querverengtes Becken höchst eigenthümlicher Art,
}

unter Vorzeigung desselben gehalten in der gynäkologischen Section der

43. Versammlung deutscher Naturforscher u. s. w. zu Innsbruck am 20. September 1869

Fon

\section{Wilhelm Lange.}

(Hierzu Tafel III.)

Ich erlaube mir, der geehrten Versammlung ein fehlerhaftes weibliches Becken vorzuzeigen, welches mich in die Nothwendigkeit, den Kaiserschnitt vorzunehmen, versetzt hat und der Beachtung der Fachgenossen nicht unwerth sein dürfte, weil es einen Complex von Anomalieen darstellt, deren jede einzelne schon nicht zu den, so zu sagen, alltäglichen Vorkommnissen gehört, durch deren Verbindung mit einander daher das Becken zu einem Mischlinge höchst eigenthümlicher Art, ja vorläufig sogar zu einem Unic um gestempelt wird.

Es stammt von einer ledigen, 34jährigen, sehr geistesschwachen und fast ganz tauben, wegen grosser Armuth ihrer Eltern und wegen eigener Erwerbsunfähigkeit seit mehreren Jahren in ein Versorgungshaus aufgenommenen Erstgeschwängerten, Namens Elisabeth W ahl, deren Mutter Folgendes über sie angab: Sie sei bis zur Hälfte ihres ersten Lebensjahres ganz gesund gewesen, dann aber von der englischen Krankheit befallen worden, in deren Folge sich die später näher anzugebenden Misstaltungen ihres Körpers eingestellt hätten, und welche Ursache gewesen sei, dass sie erst in ihrem zehnten Lebensjahre laufen gelernt habe. 
Bis dahin habe sie den weitaus grössten Theil der Zeit in der Rückenlage im Bette zugebracht, sei dabei nach und nach so „krumm" geworden, dass sie eine Zeit lang, bevor sie aufrecht einherzugehen vermochte, nur ,,auf allen Vieren" herumzukriechen im Stande gewesen sei. Erst in einem Alter von sechs Jahren habe sie etwas sprechen gelernt und das, Gehör während ihrer Krankheit, die auch einen ,eitrigen Ohrenfluss" im Gefolge gehabt, fast gänzlich verloren. Ueberdies hätten sich im Laufe ihres sechsten Lebensjahres in der Beckengegend nach und nach sieben Abscesse gebildet, welche erst im zehnten Lebensjahre des Kindes zugeheilt, nach vier Jahren aber wieder aufgebrochen seien, um sich nach 5-6 Wochen wieder, und zwar nunmehr für immer, zu schliessen. Seit dieser Zeit sei ihre Tochter gesund gewesen, auch stärker gewachsen als früher, und habe in ihrem sechzehnten Lebensjahre zum ersten Male die monatliche Reinigung bekommen, diese jedoch in der Folge ein sehr unregelmässiges Verhalten gezeigt. Ueber den letztmaligen Eintritt derselben, dann über die Zeit der zum ersten Male gefühlten Fruchtbewegungen wusste weder die Schwangere selbst, noch deren Mutter eine irgend verlässliche Angabe zu machen. Die an die letztere gestellte Frage, ob ihre Tochter als Kind, oder in ihren späteren Lebensjahren nicht irgend einer gewaltsamen Einwirkung auf ihren Körper überhaupt, oder auf ihr Rückgrath insbesondere, wie z. B. und namentlich einem Sturze von einer, wenn auch nicht beträchtlichen Höhe herab, Stössen oder Schlägen auf den Rï̈cken, besonders auf den unteren Theil desselben, ausgesetzt gewesen sei, oder öfter schwere Lasten auf dem Rücken getragen habe, wurde mit Entschiedenheit verneint.

Status praesens, 52 Tage vor dem Eintritte der Geburt.

Die Körperlänge der ziemlich gut genährten Schwangeren betrug nur $114 \mathrm{Cm}$., wovon nur $42 \mathrm{Cm}$. auf den Rumpf mit Einsehluss des Halses und des Beckens entfielen.

Der Kopf erschien im Verhältnisse zu dem ungewöhnlich kurzen Rumpfe zu gross, war aber nicht regelwidrig geformt.

Die Zähne zeigten, ausser vorhandener Caries einzelner von ihnen, keine Abnormität, namentlich keine quere Riefung.

Der Thorax war im Verhältnisse zu seiner ungewöhnlichen Kürze breit, dabei nicht in einem ungewöhnlichen Grade vorgewölbt, und liess eine abnorme Dicke der Sternalenden der Rippen nicht wahrnehmen.

Der Thoraxtheil der Wirbelsäule zeigte im Bereiche seiner oberen fünf Wirbel eine sehr geringe skoliotische Krümmung nach links, 
welche im Bereiche seiner unteren sieben Wirbel durch eine"eben so geringe gleichnamige Krümmung nach rechts compensirt war. Dabei war die etwa zwischen dem sechsten und elften Brustwirbel liegende Partie des Rückens stark ausgehöhlt, offenbar somit zugleich auch eine Lordose des siebenten, achten, neunten und zehnten Brustwirbels vorhanden, während der Lumbaltheil der Wirbelsäule eine stark in die Augen springende, jedoch nicht spitzwinkelige, sondern eine ziemlich regelmässige Bogenlinie darstellende und, nach dieser gemessen, $121 \frac{1}{2} \mathrm{Mm}$. lange Kyphose bildete, beziiglich welcher ich jedoch, um es schon jetzt zu gestehen, den Irrthum beging, dass ich sie, wenn auch nicht ganz, so doch grösstentheils, als dem, wie bei manchen halisteretischen und psendo-halisteretischen Becken, von oben nach unten stark zusammengebogenen Kreuzbeine angehörig betrachtete, also für eine Kyphosis lumbo-sacralis hielt, während sie, wie sich später bei der Betrachtung des skelettirten Beckens herausstellte, in Wirklichkeit von den beiden letzten Brust- und von sämmtlichen Lendenwirbeln, deren Zahl die gewöhnliche, gebildet wurde, mithin eine Kyphosis dorso-lumbalis war.

Im Bereiche der Hinterbacken, dann etwas unterhalb der Mitte des linken Darmbeinkammes waren alte, ziemlich breite und tiefe Narben bemerkbar.

Der Nabel war verstrichen, der Unterleib stark ausgedehnt und in einem so hohen Grade nach vorn überhängend, dass er mit seinem grössten Theile auf den Oberschenkeln aufruhte und bis nahezu an die Kniee herabreichte.

Der Grund des Uterus stand hinter dem etwas nach vorn umgebogenen Processus xyphoideus des Brustbeines.

Von Kindestheilen war links aben ein kleiner, rechts oben dagegen ein grosser zu fühlen, welcher für dea Steiss gehalten wurde. Die fötalen Herztöne waren rechts hinten in der Höhe des wegen des sehr starken Hängebauches ungewöhnlich tief stehenden Nabels am stärksten zu hören. Der Raum zwischen dem Nabel und dem oberen Rande der Schambeine betrug nur 941/2 Mm.

Die Darmbeine waren klein, das rechte hatte eine viel flachere Stellung, als das linke, ja stellte eine fast horizontale Ebene dar, daher auch sein Kamm etwas niedriger stand, als jener des anscheinend normal gestellten linken; die Entfernung ihrer vorderen oberen Stachel von einander betrug $216 \mathrm{Mm}$., der grösste Abstand ihrer Kämme von einander dagegen $243 \mathrm{Mm}$; endlich war eine Zusammenbiegung: dieser Knochen von vorn nach hinten nicht nachweisbar, hingegen etwas unterhalb der, wie es schien, ein wenig nach innen gebogenen Mitte der Crista des linken, wo die schon erwähnte alte Narbe sich befand, ein etwa exbsengrosser etwas rauher Knochenhöcker zu fühlen war. Auf jedem der beiden Darmbeinkämme lag eine Rippe auf.

Die vordere Wand des Beckens zeigte die Schambeinverbindung sehr stark schnabelförmig vorspringend und in einem so bedeutenden Grade nach oben geschoben, dass, wofür auch die unge- 
wöhnlich hohe Lage der äusseren Scham sprach, die Neigung des Beckens als sehr verringert, wenn nịcht sogar als ganz annullirt angenommen werden musste. Dabei war es mir übrigens nicht möglich, hinsichtlich der Anwesenheit oder Abwesenheit einer Einbiegung: der horizontalen Schambeinäste gegen einander vollkommen in's Klare zu kommen. Die Distantia intertrochanterica betrug nur 2021/2 Mm. und, nach der Lage der Gelenksköpfe der Oberschenkel zu schliessen, waren die Hüftgelenkspfannen mehr nach vorn, als nach den Seiten hin gerichtet.

Bei der innerlichen Untersuchung zeigte sich der Scheitel des Schambogens als ein so spitzer Winkel, dass die Spitze meines Zeigefingers nicht einmal mit ihrem Radialrande, geschweige ihrer Quere nach, in ihm Raum fand. Nach unten hin wichen die Schenkel des Schambogens so weit von einander, dass etwa an der Stelle der Verbindung der absteigenden Schambeinäste mit den aufsteigenden Aesten der Sitzbeine die Nagelglieder meines Zeige- und Mittelfingers, quer neben einander gelegt, gerade Platz fanden, ich die Weite des Schambogens an dieser Stelle daher auf $31^{1 / 2} \mathrm{Mm}$. schätzte, während. ich dieselben Fingerglieder in gleicher Lage gegen einander noch tiefer unten, nämlich unmittelbar vor dem vorderen Ende der Sitzknorren ebenso, wie zwischen diesen selbst, etwa $63 / 4 \mathrm{Mm}$. weit von einander spreitzen konnte, an diesen beiden Stellen sonach eine Weite von etwa $38^{3} / 4 \mathrm{Mm}$. anzunehmen war.

Weder mit dem Zeigefinger allein, noch mit dem ihm beigesellten Mittelfinger vermochte ich das Steiss- und Kreuzbein zu erreichen und, was mich ganz besonders befremdete, das Promontorium aufzufinden, während sich die Sitzstachel und die Böden der Hüftgelenkspfannen so nahe an einander gerückt zeigten, dass ich die Entfernung der ersteren von einander jener der Sitzknorren von einander gleich erachten, somit auf $38^{3} / 4 \mathrm{Mm}$., den Abstand der letzteren von einander dagegen auf etwa $81 \mathrm{Mm}$. schätzen zu sollen glaubte. Ebensowenig vermochte ich, mit den genannten zwei Fingern den Eingang des Beckens zu umkreisen. Es war mir daher mit ihnen auch nicht möglich, die Schambeinkämme in Beziehung auf etwa an ihnen vorhandene Scharfkantigkeit oder Stachelbildung zu prüfen, die Einführung der halben, geschweige der ganzen Hand in das Becken aber der so grossen Beschränktheit seines Ausganges halber durchaus unthunlich.

Der ein rundes Grübchen bildende äussere Muttermund stand so hoch und war so stark nach hinten gerichtet, dass auch die Länge der Vaginalportion nicht beurtheilt werden konnte. Ein vorliegender Kindestheil war nicht zu fühlen.

Die Extremitäten zeigten weder Verkrümmungen, noch eine abnorme Dicke der Epiphysen ihrer Röhrenknochen, standen jedoch hinsichtlich ihrer Länge zu dem kurzen Rumpfe in einem grossen Contraste, welcher an den oberen in einer um so auffallenderen Weise hervortrat, als, wenn dieselben frei herabhingen, die Spitzen. der Finger bis drei Querfinger weit unter die Kniee herabreichten. 
Endlich verdient noch angeftihrt zu werden, dass der Gang der Schwangeren ein eigenthümlich watschelnder war und dass dabei die Fuisse, besonders der rechte, stark nach auswärts gedreht wurden und sich gewissermaassen mit einander kreuzten.

Auf Grund der anamnestischen Erhebungen und der wiederholten Untersuchungen glaubte ich annehmen zu dürfen, dass die Schwangere an Rachitis gelitten, und dass das Becken ein rachitisches mit theilweise halisteretischer Form, als ein sogenanntes pseudohalisteretisches, anzusehen sei. Es zeigte jedoch nicht jene höchstgradige Ausprägung der halisteretischen Form, bei welcher der Eingang nur mehr eine dreiarmige Spalte bildet, sondern jenes mittleren Grades, bei welchem diese Apertur entweder noch einem Kartenherz ähnelt, dessen Spitze gegen die Schossfuge hin ausgezogen erscheint, oder aber nahezu ein Dreieck darstellt, dessen beide nach hinten liegende Winkel abgerundet sind. Dagegen war die quere Verengung des Ausganges so stark, dass schon dieser allein wegen, folglich ohne Rücksicht auf den offenbar ungleich weniger verengten Eingang, der Kaiserschnitt unbedingt angezeigt war.

Ich glaube mir es schuldig zu sein, ausdrücklich anzuführen, dass, als mir der vorliegende Fall vorkam (1857), jene Art querer Verengung des Beckens, welche zum Unterschiede von der von Robert zuerst beschriebenen, die kyphotische genannt wird, noch nicht bekannt war. Ich würde daher höchst wahrscheinlich auch dann die eben ausgesprochene Diagnose gestellt haben, wenn ich hinsichtlich des Sitzes der Kyphose einen Irrthum nicht begangen hätte, und zwar trotzdem, dass mir der einer so hochgradigen Verengung des Beckens gegenüber jedenfalls ungewöhnliche, daher immerhin auffallende Umstand nicht entgangen war, dass die Rachitis am übrigen Knochengerüste, die Wirbelsäule allein ausgenommen, keine nachweisbaren Merkmale hinterlassen hatte. Ja ich mache kein Hehl daraus, dass ich zu der Erkenntniss, das Becken sei, von seinen übrigen höchst auffallenden Anomalieen ganz abgesehen, kein pseudohalisteretisches, sondern ein kyphotisch-querverengtes, erst dann gelangt bin, als ich nicht nur die bis jetat erschienenen Arbeiten über diese erst im Jahre 1865 (durch Breis ky) genau bekannt gewordene Beckenanomalie gelesen, sondern auch das in Zürich befindliche, von Moor beschriebene Exemplar derselben gesehen hatte. Endlich glaube ich mich mit der Thatsache trösten zu können, dass selbst durch eine bis in's kleinste Detail genaue, somit auch jene später nam- 
haft zu machenden übrigen Abnormitäten umfassende Diagnose, durch welche ich bei der ersten Anschauung des skelettirten Beckens im höchsten Grade überrascht wurde, deren Erkennung an Lebenden übrigens zum grössten Theile gar nicht möglich ist, die Nothwendigkeit des Kaiserschnittes nicht abgewendet worden wäre.

Betreffs dieser in Chloroformnarkose ausgeführten Operation beschränke ich mich auf folgende Angäben:

Der Schnitt durch die vordere Bauchwand wurde in der Linea alba geführt, musste jedoch wegen der geringen Entfernung des $\mathrm{Na}$ bels vom oberen Rande der Schambeinverbindung reichlich $54 \mathrm{Mm}$. über dem ersteren begonnen werden. - Der Schnitt durch den Uterus traf die Placenta, deren nach links gerichteter Rand sofort durch die Wunde hervorgedrängt wurde. Während jeh das in dritter Schädellage (Stirn nach links vorn) befindliche Kind herausbeförderte, wurde die ganze Placenta durch die Wunde herausgepresst und fiel zwischen die Schenkel der Operirten. - Die Uteruswunde erwies sich dem Kopfe des Kindes gegenüber als zu klein und musste nach unten und oben vergrössert werden, und die Entwickelung des Kindes erforderte einen viel grösseren Zeitaufwand, als es wünschenswerth war, Nichtsdestoweniger kam das ausgetragene Mädchen von mittlerer Grösse' nur in einem sò leichten Grade asphyktisch zur Welt, dass es sebr bald zum vollen Leben gebracht werden konnte. - Der Uterus zog sich, nachdem einer ziemlich starken Blutung aus der Placentarstelle wegen von der Wunde aus einige Stückchen Eis in ihn eingebracht worden waren, in erwünschter Weise zusammen. - Während der ganzen Operation fiel nur ein Mal, und zwar links unten, eine kleine Darmschlinge vor, die sich sehr leicht zurückbringen liess. Dagegen kam es, nachdem schon die meisten Hefte angelegt waren, in Folge eines heftigen Erbrechens zu einem so bedeutenden Vorfalle der Gedärme, dass nicht nur im unteren Wundwinkel, sondern auch zwischen den einzelnen Heften grosse Schlingen sich hervordrängten, deren Reposition nicht eben leicht war und zu deren Zurückhaltung die Anlegung von mehr Heften, als deren sonst erforderlich gewesen wären, nothwendig wurde.

Ungeachtet gleich nach vollendeter Anlegung des Verbandes Eisblasen auf den Unterleib angewandt wurden, die sehr aufgeregte Operirte bald nach ihrem Erwachen aus der Narkose 0,015 Morphium bekam, ihr von Stunde zu Stunde kleine Eiswasserklystiere beigebracht wurden und sie die ihr wegen des bald sich einstellenden heftigen Durstes verordneten Eispillen mit grossem Behagen sehr fleissig zu sich nahm, tauchten sehr bald die Erscheinungen einer Perimetritis auf, welche sich mit rasender Eile zu einer allgemeinen Peritonitis mit starker Exsudation gestaltete und schon zwölf Stunden nach der Operation dem Leben der Entbundenen ein Ziel setzte. 
Ich gehe nun an die Beschreibung des Beckens, mit welchem die beiden Oberschenkelknochen, der ganze Lendentheil der Wirbelsäule, die Hälfte des elften, dann der ganze zwölfte Brustwirbel, an dem noch die letzten, ungleich langen und breiten und ungleich gebogenen falschen Rippen haften, in Verbindung gelassen worden sind, während der dazu gehörige Thorax, in Verbindung mit dem Halstheile der Wirbelsäule, getrennt in der Sammlung meiner Klinik aufbewahrt wird.

Im Ganzen betrachtet, macht das Becken vermöge der Kleinheit und Zierlichkeit seiner Knochen den Eindruck, als sei es seiner ursprünglichen Anlage nach dazu bestimmt gewesen, ein allgemein gleichmässig zu enges zu werden. Dabei sind seine Knochen ausnehmend weiss und von sehr derber Consistenz.

Im Besonderen ist Folgendes hervorzuheben:

Von den ungenannten Beinen, wenn man sie von der Mitte der Sitzknorren zum höchsten Punkte der Darmbeinkämme misst, hat das linke eine Höhe oder Länge von 1641/4 Mm., das rechte dagegen nur von $1571 / 2 \mathrm{Mm}$.

Die Hüftgelenkspfannen zeigen das schon angegebene Verhalten.

In der Nähe des hinteren Endes des rechten grossen Hüftausschnittes findet sich ein $4 \frac{1}{2} \mathrm{Mm}$. langes und an seiner Basis fast eben so breites, an seiner äusseren Fläche convexes, an der inneren concares, somit fingernagelförmiges, mit seinem freien, etwas schmäleren Rande schräg nach unten und vorn gerichtetes Osteophyt.

Von den Darmbein en liegt, wie schon angegeben worden, das rechte so flach, dass es nahezu eine horizontale Ebene bildet, und misst von seinem vorderen oberen zum hinteren oberen Stachel $123^{3 / 4} \mathrm{Mm}$., vom höchsten Punkte seines Kammes zur Mitte der Linea arcuata $67 \% / 2 \mathrm{Mm}$.; während das linke, wie gleichfalls schon angegeben worden, die normale Stellung hat und bei ihm von den genannten Maassen das erste jenem des rechten gleich ist, das zweite hingegen jenes des rechten um $6^{3 / 4} \mathrm{Mm}$. übersteigt. Die Entfernung der vorderen oberen Stachel der Knochen, dann der grösste Abstand ihrer Kämme von einander sind bereits angegeben worden. Die Schaufel des rechten Darmbeines ist an einer etwa $108 \mathrm{Mm}$. im Umfange messenden, jene des linken nur an einer halb so grossen Stelle bis zur Durchsichtigkeit verdünnt, und findet sich an der äusseren Fläche des linken an der schon früher 
bezeichneten Stelle, nämlich ein wenig unterhalb der etwas nach einwärts gebogenen Mitte seiner Crista, ein rundliches, ziemlich rauhes Osteophyt von der Grösse einer grossen Erbse. Von den hinteren oberen Darmbeinstacheln, in deren Nachbarschaft ebenso, wie an noch mehreren Stellen an der äusseren Fläche der Darmbeine, grössere und kleinere Osteophyten vorfindig sind, steht vom ersten Dornfortsatze des Kreuzbeines der rechte 291/4 Mm., der linke dagegen nur reichlich $18 \mathrm{Mm}$. ab, der rechte nebstdem zugleich um 131/2 Mm. tiefer, als der linke.

Die vorher angeführte, im Vergleiche mit jener des linken um $6^{3} / 4 \mathrm{Mm}$. geringere Höhe des rechten ungenannten Beines findet in der so flachen Stellung des ihm angehörigen Darmbeines eine eben so ungezwungene, als genügende Erklärung.

Die queren Aeste der Schambeine verlaufen gestreckt, also ohne eingeknickt oder gegen einander eingebogen zu sein, und so gegen einander convergirend nach vorn, dass sie unmittelbar hinter der Schambeinverbindung nur $15^{3 / 4} \mathrm{Mm}$. von einander abstehen, während die Entfernung ihrer hinteren Enden von einander $761 / 2 \mathrm{Mm}$. beträgt. Dabei bildet die Crista des linken eine $33^{3} / 4 \mathrm{Mm}$. lange, an ihrer breitesten Stelle nahezu $54 \mathrm{Mm}$. hohe, messerscharfe Kante, während dęr Kamm des rechten zwar auch schärfer ist, als gegewöhnlich, ohne jedoch mit Recht eine scharfe Kante genannt werden zu können.

Die Schambeinverbindung ist $31 \% \mathrm{Mm}$. hoch, bildet einen stark rorspringenden Schnabel und ist in einem so hohen Grade nach oben geschoben, dass ihr oberer Rand mit einer das hintere Ende des Beckeneinganges umgränzenden Linie in gleicher Ebene liegt, die Neigung des Beckens sonach auf Null reducirt ist. Zugleich. steht sie der Medianlinie des Kreuzbeines nicht gerade gegenüber, sondern weicht von derselben etwa $6^{3 / 4} \mathrm{Mm}$. nach links ab.

Der Schambogen beginnt an der Schambeinverbindung mit einem spitzen Winkel und erweitert sich nach abwärts allmälig nur in dem Maasse, dass seine Weite ungefähr in der Mitte desselben blos 291/4 Mm., an seinem unteren Ende 31/2 Mm. beträgt. Seine Schenkel zeigen keine Verbiegungen und der linke steht in merklicher Weise hinter dem rechten zurück.

Das Kreuzbein besteht aus fünf falschen Wirbeln, von denen jedoch der unterste bis auf seine Querfortsätze und jene Fortsätze, welche die Hörner des Kreuzbeines genannt werden, 
durch Caries zerstört ist. Sämmtliche vorhandene Kreuzbeinwirbel sind bis auf ihre Dornfortsätze so innig mit einander verschmolzen, dass von den queren Knochenleisten, welche unter normalen Verhältnissen die frühere Trennung andeuten, auch nicht eine Spur nachweisbar ist. Denkt man sich die Lücke des fehlenden Körpers des fünften Kreuzwirbels ergänzt, so beträgt die Länge des Kreuzbeines, in gerader Linie gemessen, nur 51 $\frac{1}{2} \mathrm{Mm}$., seine Breite am unteren Ende $35^{3 / 4} \mathrm{Mm}$., in der Mitte $58 \% \frac{1}{2} \mathrm{Mm}$., am oberen Ende $94 \% / 2 \mathrm{Mm}$. Seine vordere Fläche ist nur wenig ausgehöhlt, nicht rollkommen gerade nach vorn, sondern in Folge einer geringen Drehung des Knochens um seine Längenaxe ein wenig schräg nach links gerichtet, in ihrer oberen Hälfte ganz glatt, in der unteren dagegen in Folge stellenweiser, oberflächlicher cariöser Zerstörung rauh und uneben. Zugleich steht das Kreuzbein mit seiner rechten Hälfte etwas tiefer, als mit der linken. Es verläuft daher seine Längenaxe nicht gerade nach abwärts, sondern etwas schief von rechts oben nach links unten, und die Foramina sacralia, sowohl die vorderen als die hinteren, je vier Paar an Zahl und im Verhältnisse zu der ungewöhnlichen Kleinheit des Kreuzbeines von sehr ansehnlicher Umfänglichkeit, stehen rechterseits gleichfalls etwas tiefer, als linkerseits. Betrachtet man das Kreuzbein von rückwärts und fasst dabei den Sitz seiner Dornfortsätze in's Auge, so ist die linke Seitenhälfte durchaus schmäler, als die rechte, und zwar ist der Unterschied gleich der früher angegebenen Differenz des Abstandes der hinteren oberen Darmbeinstachel rom ersten Dornfortsatze des Kreuzbeines, also reichlich $11^{1 / 4} \mathrm{Mm}$. In Folge der abnormen Schmalheit der linken Kreuzbeinhälfte liegt das oberste Foramen sacrale posticum dieser Seite fast ganz hinter dem linken hinteren oberen Darmbeinstachel versteckt. Der rechte Flügel des Kreuzbeines hat eine grösste Breite von $291 / 4 \mathrm{Mm}$., der linke von nur 221/2 Mm. Während der rechte mit dem Dormbeine seiner Seite normal verbunden, ist der linke so vollständig ankylosirt, dass sich die ursprüngliche Trennung beider Knochenstücke nur angedeutet findet, und zwar im Inneren des Beckens durch einen abgerundeten, nur wenig erhabenen, glatten Knochenwulst, der in seinem Verlaufe auf der Ankylose den Verlauf des oberen Theiles des Contur des rechterseits vorhandenen Ileosacralgelenkes ziemlich getreu nachahmt, an der Aussenseite dagegen durch einen Kanal, der hinter dem hinteren oberen Darmbeinstachel, jedoch etwa $15^{3 / 4} \mathrm{Mm}$. nach aussen von ihm, in die mit einander ver- 
schmolzenen Knochenstücke in der Richtung des soeben beschriebenen $\mathrm{Knochenwulstes} \mathrm{etwa} 9 \mathrm{Mm}$. tief eindringt, blind endet, etwa den Umfang eines Gänsekiels besitzt, an seiner Eingangsmündung jedoch von oben nach unten etwas abgeplattet ist, dann durch eine Knochenleiste, welche zwischen dem hinteren unteren Darmbeinstachel und dem an ihn grenzenden Rande des Kreuzbeines fein aber scharf hervortritt, und in ihrem Verlaufe den Verlauf des entsprechenden Theiles des Contur des auf der anderen Seite vorhandenen Ileosacralgelenkes nachahmt. Wie die ganze linke Hälfte des Kreuzbeines, so steht selbstverständlich auch die ihr angehörige Ileosacralankylose etwas höher, als das Ileosacralgelenk der rechten Seite, ist aber um $13 \frac{1}{2} \mathrm{Mm}$. niedriger, als das letztere.

Das Steissbein fehlt mit alleiniger Ausnahme seines rechten Hornes und ist ohne Zweifel ebenso durch Caries zerstört worden, wie der letzte Wirbel des Kreuzbeines. Jedenfalls ist es nicht erst nachträglich abhanden gekommen, da es schon gleich nach der Herausnahme des Beckens aus der Leiche vermisst wurde. Wäre es ganz vorhanden, so würde seine Spitze vermöge des etwas schrägen Verlaufes der Längenaxe des Kreuzbeines von rechts oben nach links unten entschieden nach links hin gerichtet sein.

In Betreff der vorhandenen Kyphosis dorso-lumbalis ist noch Folgendes anzuführen:

Die Körper sämmtlicher Le ndenwirbel sind so innig mit einander ankylosirt, dass ihre vormalige Trennung vorn gar nicht, rechts und links seitlich, aber auch dies nicht bei allen, nur durch schwache transversale Knochenleisten, jene des dritten vom vierten linkerseits nebstdem durch einen nur knapp 41/2 $\mathrm{Mm}$. langen, transversalen Spalt angedeutet ist. Alle zusammen bilden daher ein Ganzes, und zwar in der Form einer Säule, deren Höhe vorn jedoch nur 221/2 Mm., hinten dagegen $45 \mathrm{Mm}$. beträgt. Von diesem Maasse kommen, insoweit es sich nach den erwähnten Knochenleisten, als Gränzmarken zwischen einigen Wirbelkörpern, bestimmen lässt, auf den ersten derselben vorn $111_{4} \mathrm{Mm}$., hinten $18 \mathrm{Mm}$; auf den zweiten und dritten zusammen vorn $6^{3 / 4} \mathrm{Mm}$, hinten $15^{3 / 4} \mathrm{Mm}$; auf den vierten und fünften zusammen vorn 41/2 Mm., hinten $11^{1 / 4} \mathrm{Mm}$. Auf ein so wahrhaft erstaunlich, ja fast unglaublich geringfügiges Maass sind die Körper sämmtlicher Lendenwirbel, am allermeisten jene des vierten und fünften 
in ihrer Höhe, während ihre Fortsätze gehörig entwickelt sind, reducirt, und zwar vorn ungleich mehr, als hinten, so dass sie mit ihrer Basis nach hinten gekehrte Keile darstellen. In demselben Verhältnisse, als die Lendenwirbelkörper in ihrer Reihenfolge voñ oben nach unten an Höhe abnehmen, wer-

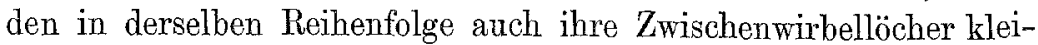
ner und die Scheidewände der letzteren immer dünner, so zwar, dass jene zwischen dem vierten und fünften dieser Löcher rechterseits nur noch Papierstärke hat, während die gleichnamige Scheidewand linkerseits sogar gänzlich verschwunden ist, hier das vierte und fünfte Intervertebralloch demnach zusammenfliessen. - Wie sämmtliche Lendenwirbelkörper mit einander, so ist der letzte derselben aber auch mit dem Kreuzbeine mittels einer vollständigen Ankylose verschmolzen, so dass dasselbe mit den Lendenwirbeln ein Ganzes bildet. Dabei ist der vordere, unter gewöhnlichen Verhältnissen bekanntlich stark vorspringende Rand der Basilarfläche des Kreuzbeines, in gleicher Weise aber auch der gleichnamige und unter gewöhnlichen Verhältnissen ebenfalls vorspringende Rand der unteren Fläche des letzten Lendenwirbels gänzlich verschwunden, von einem Promontorium demnach nicht einmal auch nur eine Andeutung vorhanden.

Während die Querfortsätze der Lendenwirbel von einander getrennt geblieben, von ihren Dornfortsätzen nur der erste und der zweite durch eine den Zwischenraum zwischen ihnen bis nahe an ihre Spitzen ausfüllende, starke und harte Knochenmasse mit einander verbunden sind und die Verbindung des Körpers des ersten Lendenwirbels mit jenem des letzten Brustwirbels normal ist, sind die Gelenksfortsätze sämmtlicher Lendenwirbel mit einander, dann die unteren des letzten mit den Gelenksfortsätzen des ersten Sacralwirbels, endlich die gleichnamigen Fortsätze der untersten zwei Brustwirbel mit einander und die unteren des letzten derselben mit den oberen des ersten Lendenwirbels ebenfalls mittels vollständiger Ankylosen verschmolzen.

An dex rechten Seitenfläche des zweiten und dritten, dann an der linken des zweiten Lendenwirbels finden sich Osteophyten, und zwar an ersterer Stelle in der Form eines transversal gestellten, dornähnlichen, an 41/2 $\mathrm{Mm}$. langen, etwa rabenfederdicken, stumpfspitzen Doppelstachels, an letzterer in der Form eines eben so gestellten und gestalteten, jedoch etwas längeren und dünneren, dabei aber spitzeren einfachen Stachels. 
In Folge ihrer kyphotischen Krümmung ragen das Lendenstück der Wirbelsäule und die letzten zwei Brustwirbel in schräger Richtung von hinten und unten nach vorn und oben so in das obere Becken herein, dass sie gleichsam eine unvollkommene Ueberdachung der grösseren hinteren Hälfte des Einganges des Beckens bilden, folglich ein ähnliches Verhalten zeigen, wie das Lendenstück der Wirbelsäule am spondylolisthetischen Becken.

Der Eingang des Beckens würde, wäre ein Promontorium vorhanden, wie jener mancher pseudohalisteretischer und wirklich hâlisteretischer Becken, ein Kartenherz mit gegen die Schambeinverbindung ausgezogener Spitze darstellen; eben des Mangels eines Promontoriums halber jedoch bildet er ein Oval, dessen im Verhältnisse zu seiner Spitze sehr breites dickeres Ende nach hinten gekehrt ist. In seinem Bereiche, und zwar an der vorderen Hälfte der Linea arcuata des rechten Darmbeines, findet sich ein Osteophyt in der Form einer $24^{3 / 4} \mathrm{Mm}$. breiten, an ihrer Oberfläche rauhen und unebenen, nach hinten als ein platter und scharfer Stachel, nach vorn als scharfe Kante etwas in ihn hineinragenden, in wagerechter Lage auf den ihren Sitz bildenden Knochen gleichsam aufgeleimten Lamelle.

Der Ausgang des Beckens stellt mehr nur eine Spalte dar, deren Breitendimensionen, insoweit der Schambogen dabei in Betracht kommt, bereits angegeben worden sind.

Von den inneren Durchmessern des Beckens beträgt

1) der gerade:

a. im Eingange $110^{3} / 4 \mathrm{Mm}$,

b. in der Beckenweite $105^{3 / 4} \mathrm{Mm}$.,

c. in der Beckenenge $112^{1 / 2} \mathrm{Mm}$.,

während derselbe im Ausgange des Mangels des Steissbeines halber nicht gemessen werden kann;

2) der quere:

a. im Eingange $99 \mathrm{Mm}$,

b. in der Beckenweite $871 / 2 \mathrm{Mm}$.,

c. in der Beckenenge $36 \mathrm{Mm}$.,

d. im Ausgange $36 \mathrm{Mm}$.;

3) der rechte schräge:

a. im Eingange $99 \mathrm{Mm}$.,

b. in der Beckenweite 101\% Mm.; 
4) der lintre schräge:

a. im Eingange $105^{3 / 4} \mathrm{Mm}$.,

b. in der Beckenweite $94^{1 / 2} \mathrm{Mm}$.,

in der Beckenenge und im Ausgange konnten beide der Abgängigkeit der Ligamenta sacro - spinosa und sacro - tuberosa halber nicht gemesen werden.

Die Diagonalconjugata misst $117 \mathrm{Mm}$.

Zieht man bei wieder hergestellter Verbindung des Thorax mit der am Becken belassenen Partie der Wirbelsäule die stellvertretende Conjugata, d. h. eine gerade Linie vom oberen Rande der Schamfuge zur Wirbelsäule, welche in der der Conjugata vera am normalen Becken entsprechenden Richtung aufsteigt, so fällt ihr hinteres Ende an den oberen Rand des Körpers des zehnten Brustwirbels. Hierbei kommt das elfte Rippenpaar auf die Darmbeinkämme zu liegen und beträgt der Raum zwischen dem oberen Rande der Schossfuge und dem unteren Ende des Schwertfortsatzes des Brustbeines nur $74^{1 / 4} \mathrm{Mm}$.

Fasst man diese Verbäitnisse in's Auge und berücksichtigt dabei zugleich die für die Insertion des Zwerchfells bestimmten Punkte, so kann man sich eine Vorstellung davon machen, wie winzig klein bei der Trägerin dieses Beckens der eigentliche Bauchhöhlenraum gewesen sein müsse, und begreifen, dass der schwangere Uterus, selbst wenn er keinen ungewöhnlich grossen Umfang erreichte, in demselben, und zwar nicht etwa erst gegen das Ende der Schwangerschaft, sondern schon viel früher, unmöglich genügenden Platz finden konnte, folglich aus ihm hervorzutreten und den vorhanden gewesenen so hochgradigen Hängebauch zu verursachen unausweichlich gezwungen war.

Soll aus der gegebenen Beschreibung des Beckens eine bis in's Einzelne gehende Diagnose construirt werden, so muss sie nach meinem Dafürhalten dahin lauten, dass das Becken als ein kyphotisch-querverengtes, zugleich rachitisches und einseitig-verengtes mit durch vollständige Ankylosen vermittelter Verschmelzung sämmtlicher Lendenwirbel mit dem Kreuzbeine zu Einem Stücke, mit vollständiger Ankylose der Gelenksfortsätzeder Lendenwirbel, des Kreuzbeines und der letzten zwei Brustwirbel, mit vollständiger linksseitiger Ileosacràlankylose, mit gänzlichem Mangel des Promontoriums, mit cariöser 
Zerstörung des letzten Kreuzwirbels bis auf seine Querfortsätze und Hörner und des Steissbeines bis auf das eine seiner Hörner, mit Ueberdachung des Einganges durch das Lendenstück der Wirbelsäule in ähnlicher Weise, wie beim spondylolisthetischen Becken, mit Scharfkantigkeit des linken queren Schambeinastes und mit mehrfacher Osteophytbildung, und zwar zumeist in der Form von Stacheln und ron Scharfkantigkeit, anzusprechen sei.

Dass übrigens, wenigstens und jedensfalls in geburtshülflichpraktischer Beziehung, unter diesen zahlreichen Anomalieen der kyphotisch-queren Verengung der erste Rang gebühre, das Becken somit in erster Linie auch als ein kyphotisch-querverengtes zu bezeichnen sei, kann, wie ich glaube, keinem $Z_{\text {weifel unterlie- }}$ gen, weil sein schädlicher Einfluss auf den Gebäract selbstverständlich ganz derselbe gewesen sein wïrde, ja hätte sein müssen, auch wenn alle übrigen Abnormitäten desselben nicht vorhanden gewesen wären, ganz abgesehen davon, dass die, von diesen zunächst in Betracht kommende einseitige Verengung schon an und für sich, um so mehr demnach im Vergleiche mit der so hochgradigen queren, ohnehin nur eine sehr geringfügige ist.

Sollte ich schliesslich meiner Ansicht über die Genese dieses in der That als ein Curiosum naturae zu betrachtenden Beckens Ausdruck geben, so würde sie möglichst kurz gefasst, folgendermaassen lauten:

Die schon nach dem ersten halben Lebensjahre des Individuums ausgebrochene Rachitis war das erste Glied in der Kette jener Vorgäuge, durch welche das Becken so verbildet wurde. Der Einfluss der Rachitis, soweit er überhaupt sich auch auf die Configuration der Becken der von ihr Ergriffenen erstreckt, wurde jedoch durch eine noch während ihrer Fortdauer hinzugetretene Entzïndung der Lendenwirbel, des Kreuzbeines und des linken Ileosacralgelenkes verhindert. Diese Entzündung, von der ja nicht wesentlichen, also als Nebensache zu betrachtenden Osteophytenbildung und stellenweisen Zerstörung einzelner Knochenpartien abgesehen, hatte die Folge, dass es zur Bildung von Ankylosen kam, und zwar einerseits zwischen den Lendenwirbeln selbst und zwischen dem letzten derselben und dem ersten Sacralwirbel, andererseits zwischen dem linken Kreuzbeinflügel und dem mit ihm verbundenen Darmbeine. Die Folge davon war Hemmung der Entwicke- 
lung, Verkümmerung und Schwund der Körper der betroffenen Wirbel und der anderen genannten Knochen. Zufällig, wenn nicht vielleicht - wer vermag dies mit Sicherheit zu bestimmen? - aus dem Grunde, weil auch die Gelenksfortsätze der mehrgenannten Wirbel ankylotisch mit einander verwuchsen, wurde von dem durch entzündliche Erweichung bedingten Schwunde der vordere Theil der Körper der erkrankten Wirbel ungleich mehr betroffen, als der hintere, den Wirbelkörpern sonach die Form von stumpfen Keilen mit nach rückwärts gekehrter Basis aufgedrungen, folglich die Nothwendigkeit, dass sie im Vereine mit einander eine Kyphose bildeten, hierdurch aber in weiterer Folge die zunächst über dieser gelegenen Brustwirbel sich zu einer compensirenden Lordose bequemen mussten, eben so unausweichlich herbeigeführt, als die einmal zu Stande gekommene Ankylose des Ileosacralgelenkes nothwendigerweise zu einseitiger Verengung des Beckens führen musste. War aber die Kyphose einmal gegeben, so konnte ihr specifischer Einfluss auf die Räumlichkeit und die Configuration des Beckens, ungeachtet die Rachitis noch fortdauerte, ebensowenig ausbleiben, als selbstverständlich die noch fortdauernde Rachitis das Zustandekommen einer einseitigen Verengung des Beckens hintanzuhalten vermochte.

Für meine Annahme, dass wirklich Rachitis im Spiele war, folglich für mein Recht, das Becken auch als rachitisches zu bezeichnen, sprechen nicht nur die Anamnese, sondern auch die skoliotischen Verkrümmungen der Wirbelsäule und gewisse Eigenthümlichkeiten des Beckens selbst, nämlich die offenbar auf gehemmte Entwickelung zurückzuführende Kleinheit und die derbe Consistenz seiner Knochen, welche allen rachitischen Becken, ferner die ausnehmend weisse Farbe derselben, welche wenigstens einer gewissen Art dieser Becken eigen ist, die stellenweise, bis zur Durchsichtigkeit gediehene Dünnheit der Darmbeinschaufeln und die bei rachitischen Becken erfahrungsgemäss am häufigsten vorkommende Scharfkantigkeit im Bereiche der Schambeinkämme. Dass ferner auch die genannten Entzündungen im Spiele waren, beweisen die vielen an dem Becken vorhandenen, im wahren Sinne des Wortes handgreiflichen Folgen derselben, nämlich die Osteophyten, die stellenweise cariöse Zerstörung der Knochen und die so zahlreichen Ankylosen. Dass ïbrigens diese Entzündungen mit der Rachitis im Causalnexus gestanden haben, halte ich nicht für unmöglich, zumal da die Einwirkung zur Hervorrufung einer Entzündung von Knochen überhaupt, jener, welche an der Bildung 
von Kyphosen der Wirbelsäule betheiligt sind, insbesondere, ein traumatischer Insult auf die Wirbelsäule, wie Fall, Schlag und Stoss auf dieselbe, sowie häufiges Tragen schwerer Lasten auf dem Rücken nicht nachgewiesen werden kann, vielmehr die Frage, ob die vormalige Besitzerin des Beckens je einer solchen Schädlichkeit ausgesetzt gewesen sei, von der Mutter derselben sogar entschieden verneint worden ist.

Dagegen nehme ich zu behaupten keinen Anstand, dass die Entzündung der Lendenwirbel und des Kreuzbeines früher, als jene des Ileosacralgelenkes, sowie überhaupt in einer sehr frühen Lebensperiode des Individuums aufgetreten sein müsse, weil die Verkümmerung der genannten Wirbel und des Kreuzbeines eine wahrhaft enorme, die einseitige Verengung des Beckens hingegen eine so geringfügige ist, dass mit vollster Sicherheit angenommen werden kann, das Becken müsse zu der Zeit, als die Ileosacralankylose als Ursache der einseitigen Verengung desselben zu Stande kam, in seiner Ausbildung schon viel weiter vorgeschritten gewesen sein, als es zu jener Zeit gewesen, in welcher die Entzündung der Lendenwirbel und des Kreuzbeines eintrat.

Ueber die Art und Weise, wie Kyphosen des unteren Abschnittes der Wirbelsäule quere Verengung des Beckens bewirken, weiss ich vor der Hand keine andere, jedenfalls keine bessere Erklärung zu geben, als sie von Anderen, nämlich von Breisky und von Moor, bereits gegeben worden ist. 


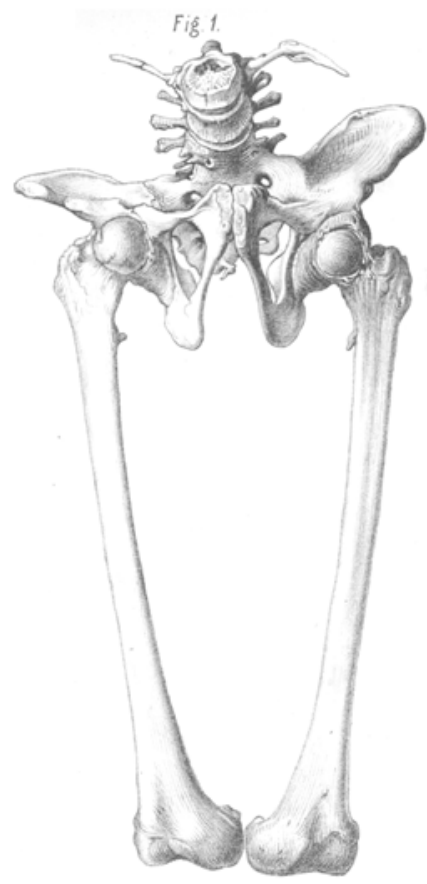

Vorderansicht.

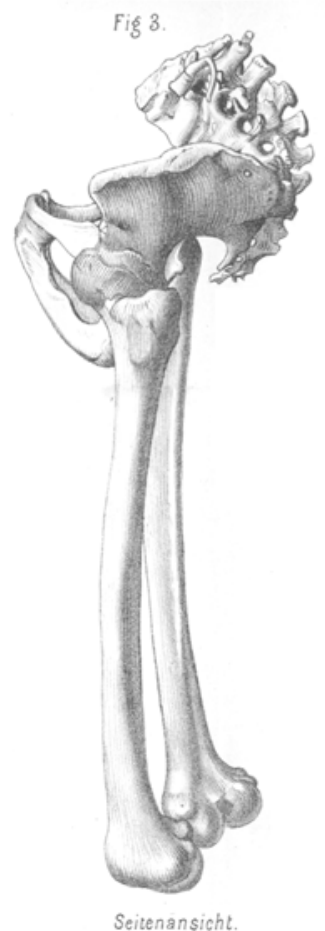

Seitenansicht.

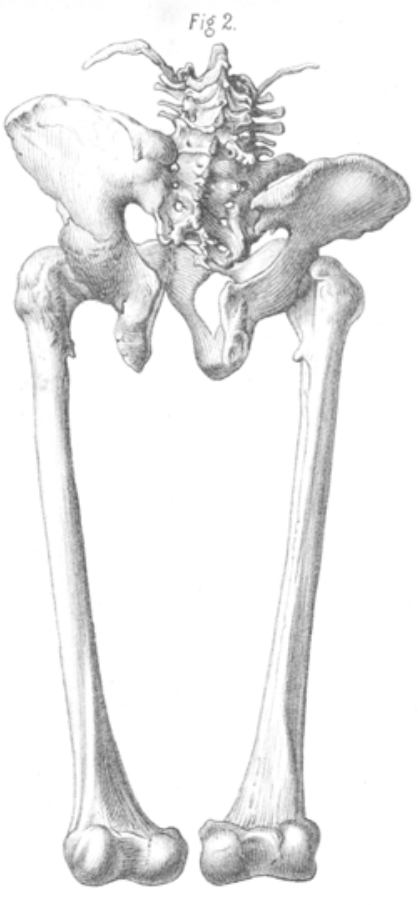

Rückansicht.

Fig 4.

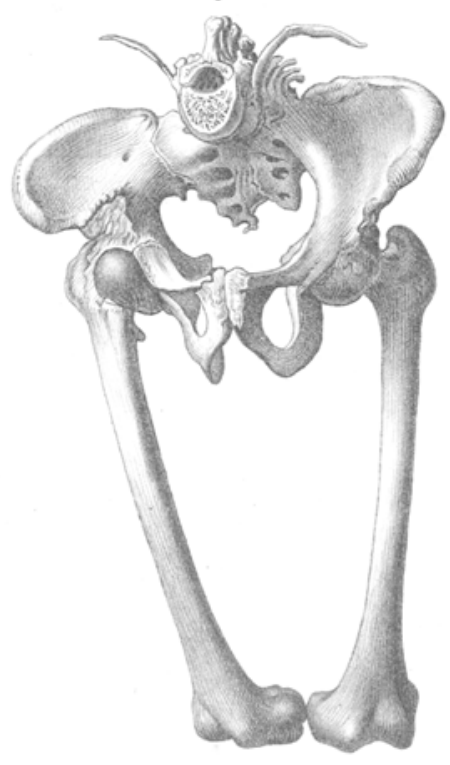

Fig 5.

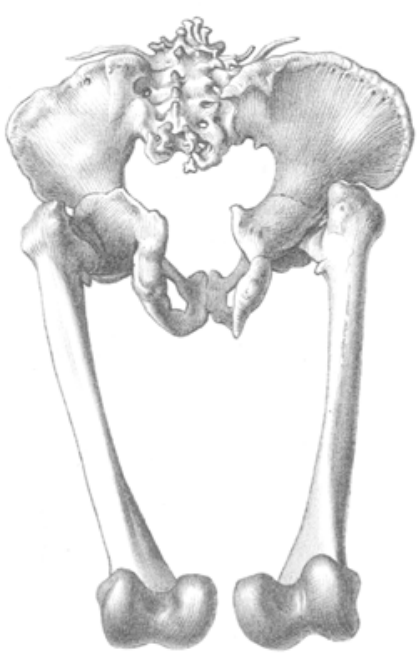

Ausgang.

Eingang. 